\title{
Comparison of Breast Cancer to Healthy Control Tissue Discovers Novel Markers with Potential for Prognosis and Early Detection
}

\author{
Michèl Schummer ${ }^{1 *}$, Ann Green ${ }^{1}$, J. David Beatty ${ }^{2}$, Beth Y. Karlan ${ }^{3}$, Scott Karlan ${ }^{4}$, Jenny Gross ${ }^{3}$, Sean \\ Thornton ${ }^{5}$, Martin Mclntosh ${ }^{1}$, Nicole Urban ${ }^{1}$
}

1 Molecular Diagnostics Program, Fred Hutchinson Cancer Research Center, Seattle, Washington, United States of America, 2 Breast Surgical Clinic, Swedish Cancer Institute, Seattle, Washington, United States of America, $\mathbf{3}$ Women's Cancer Research Institute at the Samuel Oschin Comprehensive Cancer Institute, Cedars-Sinai Medical Center, Los Angeles, California, United States of America, $\mathbf{4}$ Saul and Joyce Brandman Breast Center, Cedars-Sinai Medical Center, Los Angeles, California, United States of America, $\mathbf{5}$ Cellnetix Pathology and Laboratories, Seattle, Washington, United States of America

\begin{abstract}
This study was initiated to identify biomarkers with potential value for the early detection of poor-outcome breast cancer. Two sets of well-characterized tissues were utilized: one from breast cancer patients with favorable vs. poor outcome and the other from healthy women undergoing reduction mammaplasty. Over 46 differentially expressed genes were identified from a large list of potential targets by a) mining publicly available expression data (identifying 134 genes for quantitative PCR) and b) utilizing a commercial PCR array. Three genes show elevated expression in cancers with poor outcome and low expression in all other tissues, warranting further investigation as potential blood markers for early detection of cancers with poor outcome. Twelve genes showed lower expression in cancers with poor outcome than in cancers with favorable outcome but no differential expression between aggressive cancers and most healthy controls. These genes are more likely to be useful as prognostic tissue markers than as serum markers for early detection of aggressive disease. As a secondary finding was that when histologically normal breast tissue was removed from a distant site in a breast with cancer, 7 of 38 specimens displayed a cancer-like expression profile, while the remaining 31 were genetically similar to the reduction mammaplasty control group. This finding suggests that some regions of ipsilateral histologically 'normal' breast tissue are predisposed to becoming malignant and that normal-appearing tissue with malignant signature might warrant treatment to prevent new primary tumors.
\end{abstract}

Citation: Schummer M, Green A, Beatty JD, Karlan BY, Karlan S, et al. (2010) Comparison of Breast Cancer to Healthy Control Tissue Discovers Novel Markers with Potential for Prognosis and Early Detection. PLoS ONE 5(2): e9122. doi:10.1371/journal.pone.0009122

Editor: Kerstin Borgmann, University Medical Center Hamburg-Eppendorf, Germany

Received October 24, 2009; Accepted January 15, 2010; Published February 9, 2010

Copyright: (c) 2010 Schummer et al. This is an open-access article distributed under the terms of the Creative Commons Attribution License, which permits unrestricted use, distribution, and reproduction in any medium, provided the original author and source are credited.

Funding: Work was supported by Department of Defense's Congressionally Directed Medical Research Programs' DAMD 17-02-1-0691 and by NCl/Avon Grant P50 CA083636. Sean Thornton in employed by Cellnetix. The funders, including Cellnetix, had no role in study design, data collection and analysis, decision to publish, or preparation of the manuscript.

Competing Interests: Dr. Sean Thornton is participating in this research without monetary remuneration. The specimens from which the sample tissues are collectd are those for which his company, Cellnetix, is contracted indirectly to Swedish Medical Center to evaluate for clinical (medical) reasons for direct patient treatment. His company is paid for their usual patient care roles through normal (i.e., insurance and other payer) channels. No funding of the research comes from Cellnetix, Swedish Hospital or from private persons. His only compensation was through his usual and customary salary at Cellnetix. Cellnetix has no competing products, is not engaged in product development, or patenting any technology relating to this research. This does therefore not alter the author's adherence to all the PLoS policies on sharing data and materials.

*E-mail: mschumme@fhcrc.org

\section{Introduction}

Our goal in this study was to identify biomarkers with potential value for the early detection of poor-outcome breast cancer. In 1999 we successfully used a transcript-based discovery approach to identify early detection markers for ovarian cancer [1]. Following the 5 phases of screening biomarker development proposed by Pepe et al. [2], HE4, the product of the human epididymis gene WFDC2, was developed into a serum assay [3,4] that is now approved for remission monitoring of ovarian cancer. It is being evaluated for its potential role in screening and is considered a successful product of translational biomarker research.

Because breast cancer marker research has focused mainly on prognosis [5], there are few comprehensive studies to identify early detection markers. We therefore relied on our previously successful approach using gene discovery by cDNA microarray followed by expression validation through polymerase chain reaction (PCR), ranking of potential markers and the development and testing of serum assays. For breast cancer, a large body of research already available in the public domain allowed us to forego our own microarray work; instead we mined publicly available expression data in tissues of breast cancer and normal healthy controls.

One of the lessons learned from previous gene discovery experiments is the importance of having high-quality appropriately preserved specimens and matching patient data. We therefore spent considerable effort on the accrual of needed tissues. In close collaboration with participating surgeons and pathologists, we were able to collect specimens in the operating and gross rooms where they were processed with as little delay as possible, thus minimizing variability. In addition, routine clinical gross and microscopic tissue analysis was complemented with routine research histological examination on the actual tissue piece that was later used for expression analysis. Breast tissues from breast cancer patients were then compared to those from healthy 
individuals. While normal tissue adjacent to the cancer is relatively easy to obtain, we feared that in cancer patients, cancer-related pathways may be perturbed in these tissues [6]. We therefore used normal tissue from breast reduction mammaplasties as controls.

We identified a few genes meeting necessary criteria for potential development as serum markers for diagnosis or early detection of poor-outcome breast cancer. In addition we identified several genes with potential to guide prognosis and therapy.

\section{Results and Discussion}

Our goal was to identify tissue markers that differentiate between healthy breast tissues and breast cancer tissue from patients with poor outcome. This was done in a series of successive steps: Establishment of the range of expression of genes in healthy breast tissue; database mining to identify potential genes with differential expression between breast cancers and controls; expression validation of these genes in breast cancer tissues and controls; and identification of those genes with over-expression in cancers with poor outcome relative to both good-outcome cancer and healthy control tissue.

\section{Determination of Components of Variation of Normal Tissue}

The primary goal was to identify genes that discriminate between normal breast tissue and invasive carcinoma of the breast. Since normal breast tissue was to be used as a reference, a threshold needed to be determined above which a gene would be labeled as differentially expressed. Therefore we assessed the variability in gene expression within normal breast tissue To our knowledge, this has not previously been well studied. Quantitative PCR (SYBR) expression analysis was performed for 18 genes on an average of 3.5 tissue slices per breast from 10 women with bilateral reduction mammaplasty. The PCRs were normalized by their median and the duplicate runs were averaged. Table 1 shows overall gene expression variability (as standard deviation) and which fraction of it is attributable to the component variabilities of woman-to-woman (averaging 64\% $\pm 9 \%$ ), within-breast (averaging $30 \% \pm 9 \%$ ) and left-to-right breast (averaging 6\% $63 \%$ ). These percentages represent the overall magnitude of the different sources of variation as determined by ANOVA analyses. As expected, between-woman variability is greatest, twice that of within-breast variability, implying that the largest source of variation is heterogeneity among women. Approximately $30 \%$ of overall variation was explained by variation in the molecular behavior of different tissue specimens of the same breast (note the assays were performed in duplicate, and the coefficient of variation $(\mathrm{CV})$ of the assay was small compared to each of these components of variation and so can be ignored). The smallest source of variation is the between-breast component, implying that normal material from a contralateral breast is a good surrogate for normal material from the affected breast. Note that the $30 \%$ within- and $6 \%$ between-breast variation does not imply that two biopsies from different breasts are more similar than two biopsies from the same

Table 1. Variability in gene expression.

\begin{tabular}{|c|c|c|c|c|}
\hline \multirow[b]{2}{*}{ Marker } & \multirow[b]{2}{*}{ StDev } & \multicolumn{3}{|c|}{ Proportion of Standard Deviation } \\
\hline & & Between women & Within breast & $\begin{array}{l}\text { Between breasts (same } \\
\text { woman) }\end{array}$ \\
\hline ASPN & 0.88 & $75 \%$ & $21 \%$ & $3 \%$ \\
\hline CAV1 & 0.89 & $60 \%$ & $38 \%$ & $2 \%$ \\
\hline CFB & 0.74 & $46 \%$ & $46 \%$ & $8 \%$ \\
\hline COL1A2 & 1.13 & $76 \%$ & $15 \%$ & $10 \%$ \\
\hline CTGF & 1.07 & $67 \%$ & $28 \%$ & $6 \%$ \\
\hline GATA3 & 1.61 & $60 \%$ & $36 \%$ & $4 \%$ \\
\hline LETMD1 & 0.46 & $79 \%$ & $11 \%$ & $9 \%$ \\
\hline MGST1 & 0.94 & $59 \%$ & $37 \%$ & $4 \%$ \\
\hline LYZ & 1.08 & $66 \%$ & $23 \%$ & $8 \%$ \\
\hline MMP2 & 0.63 & $50 \%$ & $39 \%$ & $10 \%$ \\
\hline MUC1 & 2.34 & $62 \%$ & $34 \%$ & $4 \%$ \\
\hline SPARC & 0.71 & $74 \%$ & $22 \%$ & $3 \%$ \\
\hline SUMF2 & 0.39 & $55 \%$ & $35 \%$ & $7 \%$ \\
\hline TIMP1 & 0.69 & $65 \%$ & $26 \%$ & $9 \%$ \\
\hline TIMP2 & 0.49 & $63 \%$ & $29 \%$ & $7 \%$ \\
\hline TIMP3 & 0.60 & $67 \%$ & $30 \%$ & $3 \%$ \\
\hline WFDC2 & 2.73 & $61 \%$ & $35 \%$ & $4 \%$ \\
\hline \multirow[t]{3}{*}{ YWHAZ } & 0.37 & $64 \%$ & $30 \%$ & $5 \%$ \\
\hline & Mean & $64 \%$ & $30 \%$ & $6 \%$ \\
\hline & StDev & $9 \%$ & $9 \%$ & $3 \%$ \\
\hline
\end{tabular}


breast. Rather, informally, a histogram of expression from biopsies across breasts will have a standard deviation that is only $6 \%$ larger than the histogram within a breast (the $6 \%$ refers to the excess variation when sampling across breasts). Because each value was median-centered, the standard deviation also represents a surrogate for $\mathrm{CV}$ in the population; it is scaled to the typical expression levels. Six of the 18 genes have standard deviations above 1 (COL1A2, CTGF, GATA3, LYZ, MUC1 and WFDC2) that could be related to a spotty expression pattern (e.g. only a few cells in a tissue express the transcript). Of note, this elevated variability is unrelated to within-breast variability. For WFDC2, the gene with the highest variability across all tissue specimens, the standard deviation is 2.73. Therefore, for subsequent comparisons of expression in cancer to that in healthy normal tissue, a threshold will be chosen consisting of the mean expression in healthy breast mammaplasty tissue plus 3 standard deviations (viz. 8.19 for WFDC2). The same calculation will be done for each gene.

\section{Database Mining Identifies Genes with Differential} Expression between Breast Cancer and Normal Tissues

Despite a large body of research on gene and protein expression in breast cancer, few studies include healthy controls. In those that do $[7,8,9,10,11,12,13,14,15,16]$, the control tissues are often not well characterized. Most publications report expression only in breast cancer and not in healthy controls. However, these are still useful for a cancer-to-normal comparison since their data can potentially be matched with those from other sources using healthy normal tissues. A sizable number of these breast publications use large-scale analysis, such as microarrays. These and additional expression data were compiled in a database (LevelsDB) that was then mined starting with genes contained in breast cancer data sets (4405 genes), followed by removal of genes and proteins whose subcellular localization makes the protein unlikely to be found in the blood stream by non-necrotic processes (confirmed nuclear, mitochondrial and ribosomal expression), leaving 3271. In a next step, housekeeping genes were excluded, followed by removal of genes with high levels of expression in normal tissues of organs with high cardiac output $[17,18,19]$ (expression greater than 10 times the median in bone, brain, heart, kidney, lung, liver, skeletal muscle and pancreas). We rationalize that proteins can potentially be produced at base level in many cells and that big organs (with high cardiac output) may contribute more than others to a basic blood protein level. The higher a basic blood protein level, the harder it is to detect the excess protein made by the developing tumor. From the remaining 1290 genes and proteins we retained those with low expression in normal tissues. The lack of truly normal breast expression data, except for data from one normal tissue coming from a breast with cancer [20] required the use of other normal, especially epithelial tissues for subtractive comparison, contained in six datasets in LevelsDB. The last reduction step resulted in 150 genes and proteins of which 44 were likely to be secreted or membrane bound which makes them ideal as a blood marker. These were augmented by 90 genes for which literature review suggests a potential role as breast cancer markers, resulting in 134 genes (Table 2) for subsequent expression analysis by PCR. References for these additional genes are listed in the supplemental Document S1.

\section{Expression Validation Results in 46 Differentially Expressed Genes}

To identify from the $\mathbf{1 3 4}$ genes those with the ability to discriminate between normal and malignant breast tissue, PCR expression analysis was performed on 93 tissues (24 invasive
Table 2. 71 of 126 genes discriminate cancers from controls.

\begin{tabular}{|c|c|c|c|c|c|c|c|}
\hline ADAM 12* & & CSNK2A1 & & MIF & & SCGB2A 1 & \\
\hline AGR2* & & CTGF & v & MMP1 & $\Delta$ & SCUBE2* & V \\
\hline AKT1 & & CTHRC1* & $\Delta$ & MMP10 & $\Delta$ & SDC1 & \\
\hline AMBP* & & CYP4B1* & v & MMP11 & $\Delta$ & SFRP1 & V \\
\hline ANGPT2* & V & CYR61 & v & MMP12 & $\Delta$ & SFRP2* & \\
\hline APOL1* & v & DEFA1 & & MMP13 & $\Delta$ & SNIP & \\
\hline AR & v & DEFA3 & & MMP14 & & SPARC & V \\
\hline ASPN* & $\Delta$ & ECM1* & & MMP16 & & SPP1 & $\Delta$ \\
\hline BGN* & $\Delta$ & EGFR & V & MMP2 & V & STC2* & \\
\hline BIRC5 & $\Delta$ & EPO & & MMP20 & & SUMF2* & \\
\hline BRCA2 & & EPOR & V & MMP3 & & THBS2 & \\
\hline BRMS1 & & ERBB3* & & MMP7 & V & TIMP1 & $\Delta$ \\
\hline BUB1 & $\Delta$ & ERBB4 & v & MMP8 & & TIMP2 & V \\
\hline C18orf8* & & ESR1 & $\Delta$ & MMP9 & & TIMP3 & V \\
\hline CALB2 & & ETAA1* & v & MSLN & & TIMP4 & V \\
\hline CAV1 & v & FGFR2* & v & MUC1 & $\Delta$ & TK1 & $\Delta$ \\
\hline CCNE1 & $\Delta$ & FN1* & $\Delta$ & NES & v & TM9SF2* & \\
\hline CD274 & $\Delta$ & FOXA1 & $\Delta$ & OAS1* & & TNFRSF10B & V \\
\hline CD44 & v & GATA3 & $\Delta$ & OAS2* & $\Delta$ & TNN & v \\
\hline CDH1 & & GDF15 & & PEBP1 & v & TOP2A & $\Delta$ \\
\hline CDKN1B* & v & HOXB7 & v & PEBP4 & & TP53 & \\
\hline CDX2 & & IFIT1* & & PGR & v & TRPS1 & \\
\hline CFB* & $\Delta$ & IGF2 & & PIK3CA & & TTF1 & \\
\hline COL11A1* & $\Delta$ & KRT20 & & PIP & & VCAN* & $\Delta$ \\
\hline COL1A1* & $\Delta$ & KRT7 & & PLAUR & $\Delta$ & VEGFA & \\
\hline COL1A2* & $\Delta$ & LCN2 & & PRLR* & & VTCN1 & \\
\hline COL3A1* & & LETMD 1 & v & PROCR & v & WFDC2 & $\Delta$ \\
\hline COL5A1* & $\Delta$ & LPAR3 & & PSMA5* & & WT1 & $\Delta$ \\
\hline COL5A2* & v & LRRC15* & $\Delta$ & PTPN1* & & XBP1 & \\
\hline COL6A3* & & LTF* & & PVRL4 & & YWHAZ & \\
\hline COL8A 1* & & LYZ* & & S100A7 & $\Delta$ & & \\
\hline COMP* & $\Delta$ & MGST1 & v & S100B* & v & & \\
\hline
\end{tabular}

126 genes found by mining of expression data and/or LevelsDB (asterisk). Thresholds for cancers and controls were determined by expression in the 28 normal mammaplasty tissues as mean +3 SD for genes with over-expression in the cancers and as below the minimum for genes with under-expression in the cancers. Over- $(\Delta)$ or under- (V) expression in cancer tissue with $\geq 20 \%$ of the cancers and $\leq 5 \%$ of the controls above or below threshold.

doi:10.1371/journal.pone.0009122.t002

cancers, 38 ipsilateral normals, 3 contralateral normals, 28 tissues from breast reduction surgery) from 64 women (Supplemental Tables S1 and S2). The cDNA was oligo-dT primed. A commadelimited file with the expression data is available in the supplemental document S2. PCR results for 8 genes were not conclusive even after 2 repeats and the genes were removed from further analysis (supplemental Table S3). Of the 126 remaining genes, 67 discriminated between the 25 cancer tissues and the 28 mammaplasty controls with $\geq 20 \%$ of the cancers and $\leq 5 \%$ of the controls above or below threshold (Table 2, and supplemental Table S3).

After completion of the PGR work, a new PCR-based technology (OpenArray by BioTrove, Woburn, MA) had come to the market that allowed for more rapid gene expression analysis [21]. This technology was used to confirm the expression of a 
subset of the genes on a subset of the tissues. BioTrove's Cancer Pathways OpenArray plate included primer pairs specific for 606 genes associated with DNA repair, angiogenesis, cell adhesion, apoptosis, cell cycle and many genes encoding kinases. Of the 134 genes from database mining, 41 overlapped with the OpenArray panel which was suitable for confirmation of about $30 \%$ of the original results. Out of the 94 tissues that were originally tested, 13 were randomly selected from the 24 cancer tissues and likewise 9 from the 28 mammaplasty controls. Applying the same criteria as for the original set, the OpenArray analysis of the reduced set selected the same differentially expressed genes as the analysis of the original set (supplemental Table S4). Both amplifications had good correlation (supplemental Table S5) with an averaged coefficient of variation of $38 \%(12 \%-71 \%)$, even considering the differences between both methods (oligo-dT versus random priming; differences in primer sequences).

Unsupervised cluster analysis of the 93 tissues and 67 genes shows that 46 genes have the power to separate cancer tissues from controls, thus confirming the validity of our approach (Figure 1, red and green bars in the left). The mammaplasty control patients, seen in Los Angeles, were on average 15 years younger than the patients with tumor or ipsi- and contralateral normal tissue, seen in Seattle. While differences in institution and age could potentially introduce bias, the interspersing of the normal tissues in the control cluster suggests that this is not the case (Figure 1). This can be attributed in part to the strict adherence to identical specimen collection protocols at both sites. No clustering behavior was found based on other factors listed in the supplemental Table $\mathrm{S} 1$, including mammography (BI-RADS) score and breast density at the last mammogram before diagnosis, lymph node positivity, tumor size, number of foci, stage and grade.

This first outcome from our analysis was 46 genes that show differential expression between cancers and healthy breast tissues. Having applied a similar strategy to discover serum markers for ovarian cancer $([1,2,3,4])$, we have reason to believe that these 46 genes have similar potential because our mining procedure favored proteins with expression outside of the cell and with low potential of being secreted at high level by other healthy tissue. Once proven to be differentially expressed as serum proteins, it will need to be investigated whether a marker could complement mammography in early detection.

\section{Comparison to Previously Published Results}

Comparing the present gene expression results to those previously published is difficult because prior studies rarely used healthy normal controls. While the terms "over-" and "underexpression" are common in the breast cancer literature, they most often refer to expression of one cancer state relative to another or to a cell line, and not relative to a healthy normal control. The three breast cancer publications that used mammaplasty tissue as controls confirm the expression pattern of the metalloproteinases [22], YWHAZ [6], ERBB3 and ERBB4 [23]. Furthermore COL1A1 and COL1A2 over-expression was also seen in a meta-analysis of 13 publications comparing breast cancer to largely undefined and probably ipsilateral normal tissue [24]. This gives credence to the observed expression pattern of the remaining genes.

\section{Identification of Additional Differentially Expressed Genes}

The OpenArray Cancer Pathways chip contained 606 genes of which 41 were used for confirmation of the PCR results. Unsupervised cluster analysis of the remaining 565 genes in the 13 cancers and 9 control tissue results in a clear distinction between over- and under-expressed genes. The OpenArray PCR was not duplicated and thus the results have greater error margins. Therefore more stringent filtering conditions were applied than for the original PCR: genes with differential expression in more than $30 \%$ of the tumors at a tumor-to-normal ratio of 1.2 were removed from the dataset. Of the resulting 102 genes, 88\% were found to be under-expressed in the tumor tissues (see supplemental Figure $\mathrm{S} 1$ ), as indicated by the negative $\% \mathrm{CV}$ numbers in Table 3 . Once confirmed in their expression, it is likely that some of these 102 genes will be added to the 46 potential markers.

The high number of under-expressed genes contrasts sharply with above results where over- and under-expressed genes are equally represented. The Cancer Pathways genes had been selected based on general cancer literature which includes a large number of within-cancer and cell line experiments. Our data mining on the other hand focused on breast cancer, normal-tocancer differences and extracellular expression. Hence, the former contains a larger number of intracellular, regulatory proteins. Interestingly, the differentially expressed genes in both sets are enriched for connective tissue genes, suggesting that alteration in the composition of the connective tissue is an important factor in cancer formation.

\section{Identification of Markers for Cancers with Poor Outcome}

Unsupervised cluster analysis of the original PCR data resulted in a cancer cluster with two sub-clusters, one enriched for patients with cancers of the luminal subtype and one of the basal subtype, as defined by hormone receptor and HER2 expression [25] (Figure 1). Removing the normal tissues from analysis does not alter this clustering behavior (Supplemental Figure S2). The composition of these two sub-clusters is summarized in supplemental Table S6. The luminal-like sub-cluster is defined by overexpression of the luminal markers ESR1, PGR and GATA3 $[26,27,28]$ in all of its tissues and by the over-expression of $C T G F$, $M M P 2, A R, C F B, C D 44, E P O R, C D K N 1 B, E T A A 1, F G F R 2$, TNFRSF10B, ERBB4, SCUBE2, FOXA1 and MUC1 in tissues from cancers with lobular or mixed ductal-lobular histology. Except for AR [29] and ESR1 [30], none of these genes can be linked to lobular cancer histology, in particular the comparison by Zhao et al. of ductal and lobular carcinomas [31]. The role of GATA3 for the maintenance of the luminal phenotype has been reviewed by Tlsty, particularly the correlation of low expression of GATA3 and low estrogen receptor alpha [32] which the present data confirm. The basal-like sub-cluster, characterized by the under-expression of these genes, is enriched for triple-negative (hormone receptor and HER2-negative) cancers and contains all cancer tissues of patients that are deceased (black dots) or have recurred (orange dots). Lobular breast carcinomas are known to be associated with better survival than ductal carcinomas [30,33] and triple-negative breast cancers have been associated with poor prognosis [34]. Also, in a meta-analysis of published breast cancer cDNA data, low GATA3 expression is linked with poor clinical outcome [35]. The difference between these cancer sub-clusters could therefore be attributed to the aggressiveness of the disease. Supporting this idea is the fact that the one lobular cancer tissue in the basal-enriched cluster comes from a deceased patient (patient 26), which would infer that severity of outcome supersedes histology.

The genes with over-expression in cancers with favorable as opposed to those with poor outcome are listed in Figure 1 under "mixed expression" on the left. While some of these genes have been linked to the basal subtype $[7,10]$ and some are now being used to predict disease outcome, including SCUBE2 in Oncotype DX [36] and Mammaprint [37], the majority of them may constitute a novel group of genes that predict outcome and/or 


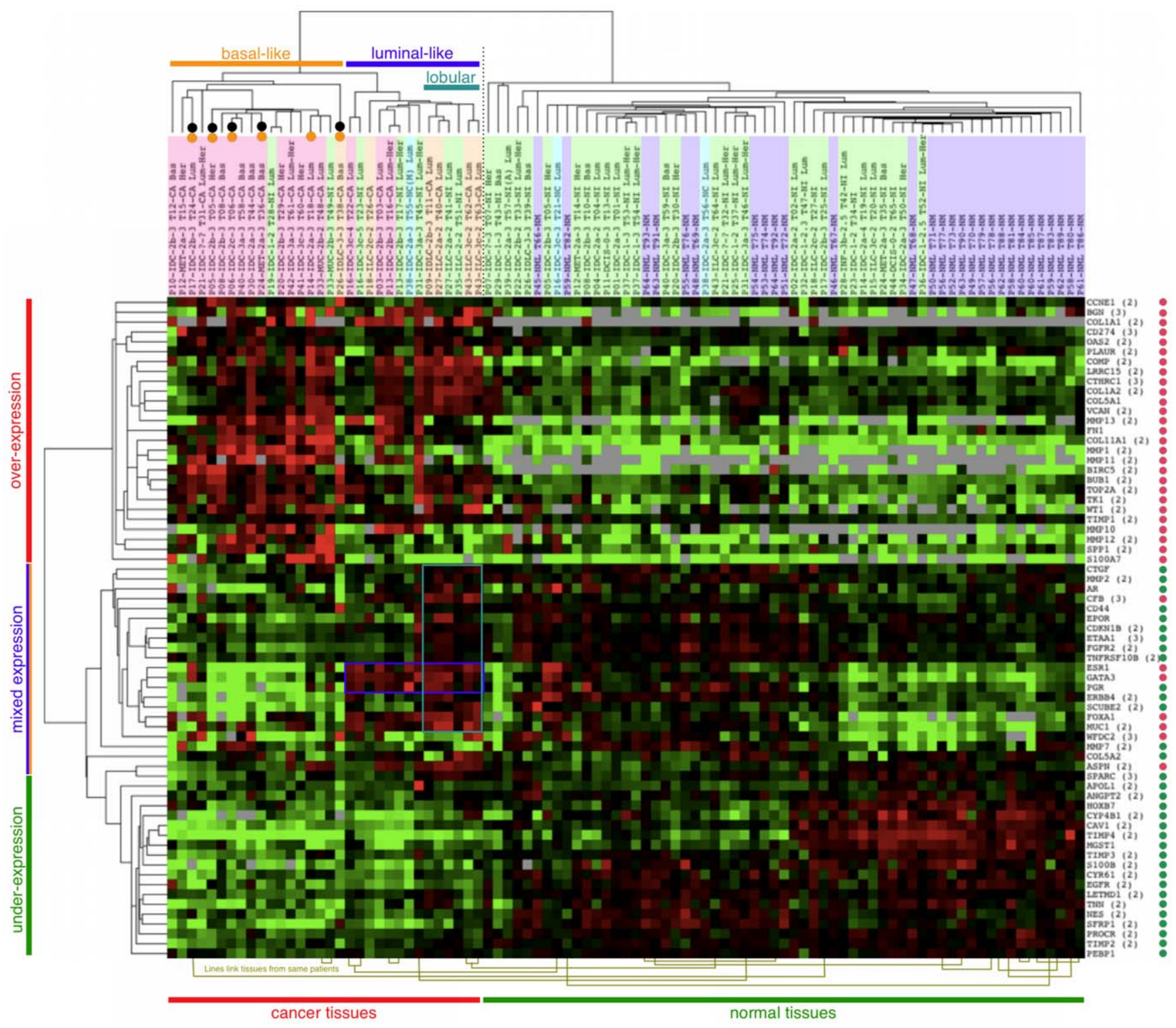

Figure 1. Unsupervised hierarchical clustering of 93 tissues and 67 genes. Unsupervised hierarchical clustering of 93 tissues (24 invasive cancers, 38 ipsilateral normal, 3 contralateral normal, 28 normal tissues from reduction mammaplasty) from 64 patients and 67 genes that discriminate between invasive tissues and mammaplasty normal tissues (red and green dots: over- and under-expression by PCR). Columns: tissues form two distinct clusters (indicated below the figure). Rows: genes form a cancer and a normal cluster, the latter being divided in one with underexpression in all cancer tissues (left, green line) and one with mixed expression (orange-blue line). Luminal-like and basal-like clusters are indicated above the figure. The part of the heat map driving the luminal-like cluster is boxed (blue: luminal-like genes, turquoise: lobular tissues). Tissues from deceased or recurred patients have a black or orange dot above the tissue descriptor which has the following abbreviated components: PatientNo Diagnosis (IDC = invasive ductal carcinoma, ILC=invasive lobular carcinoma, MET=metaplastic carcinoma, MUC=mucinous carcinoma, $\mathrm{NML}=$ normal $)$ - Stage - Grade TissueNo - Description $(\mathrm{CA}=$ cancer, $\mathrm{NM}=$ normal mammaplasty, $\mathrm{NI}=$ normal ipsilateral, $\mathrm{NC}=$ normal contralateral) BI-RADS Density Subtype (LUM = luminal, BAS = basal HER2). The tissue descriptors are shaded as follows: orange =lobular cancers, pink=other cancers, green $=$ ipsilateral normals, blue $=$ contralateral normals, purple $=$ mammaplasty normals. Heat map: Red $=$ up-regulation, green $=$ downregulation, grey $=$ missing or zero value. The lines below the heat map connect tissues from the same patient.

doi:10.1371/journal.pone.0009122.g001

inform treatment. Three genes (S100A7, SPP1 and MMP12) are over-expressed in the cancers with poor outcome compared to both cancers with favorable outcome and healthy controls warranting further investigation as potential blood markers for early detection of cancers with poor outcome.

In summary, the majority of the genes that distinguish cancers by outcome are expressed at lower levels in tissues with poor than in those with favorable outcome. Interestingly, these same genes are also under-expressed in most controls, suggesting that, by and large, molecular profiles might not be able to distinguish aggressive breast cancer from healthy breast tissue. This suggests that these genes have no potential as markers for the detection of aggressive breast cancer, but they hold potential as prognostic markers.

Histologically Normal Tissues from an Affected Breast Can Demonstrate Molecular Predisposition to Cancer

Unsupervised cluster analysis of the original PCR data placed 7 of the 38 ipsilateral normal tissues in the cancer cluster (Figure 1). 
Table 3. Genes resulting from the OpenArray analysis.

\begin{tabular}{|c|c|c|c|c|c|c|c|}
\hline CSF1 & $-100 \%$ & HADHA & $-77 \%$ & TP5313 & $-62 \%$ & SLC2A3 & $-38 \%$ \\
\hline EGR1 & $-100 \%$ & MCC & $-77 \%$ & ANPEP & $-54 \%$ & SMPD1 & $-38 \%$ \\
\hline FLT1 & $-100 \%$ & RELA & $-77 \%$ & BAG1 & $-54 \%$ & TGFBI & $-38 \%$ \\
\hline FOS & $-100 \%$ & BTG2 & $-69 \%$ & ILK & $-54 \%$ & BNIP3 & $-31 \%$ \\
\hline NID1 & $-100 \%$ & CNBP & $-69 \%$ & ING1 & $-54 \%$ & CBLB & $-31 \%$ \\
\hline SEPP1 & $-100 \%$ & DHX8 & $-69 \%$ & PECAM1 & $-54 \%$ & DEGS1 & $-31 \%$ \\
\hline SRPX & $-100 \%$ & EPHA2 & $-69 \%$ & PIR & $-54 \%$ & EGLN1 & $-31 \%$ \\
\hline TGFBR2 & $-100 \%$ & GNB2L1 & $-69 \%$ & RIPK1 & $-54 \%$ & ETV6 & $-31 \%$ \\
\hline TGFBR3 & $-100 \%$ & IGFBP4 & $-69 \%$ & SFRS7 & $-54 \%$ & FOSL2 & $-31 \%$ \\
\hline TIE1 & $-100 \%$ & NDRG1 & $-69 \%$ & TSG101 & $-54 \%$ & LDHA & $-31 \%$ \\
\hline VIM & $-100 \%$ & PAQR3 & $-69 \%$ & CAPNS1 & $-46 \%$ & NR1D1 & $-31 \%$ \\
\hline HYAL1 & $-92 \%$ & PEA15 & $-69 \%$ & CHPT1 & $-46 \%$ & PRKCD & $-31 \%$ \\
\hline PPARG & $-92 \%$ & PFDN5 & $-69 \%$ & EIF5 & $-46 \%$ & PRNP & $-31 \%$ \\
\hline RAB5A & $-92 \%$ & RAF1 & $-69 \%$ & GTF2I & $-46 \%$ & SORT1 & $-31 \%$ \\
\hline SEMA3C & $-92 \%$ & RAP1A & $-69 \%$ & JAK1 & $-46 \%$ & TRADD & $-31 \%$ \\
\hline SPRY2 & $-92 \%$ & SKI & $-69 \%$ & MDM2 & $-46 \%$ & EVL & $31 \%$ \\
\hline CCND3 & $-85 \%$ & SP1 & $-69 \%$ & MLLT10 & $-46 \%$ & HSPB1 & $31 \%$ \\
\hline CDC42BPA & $-85 \%$ & STK3 & $-69 \%$ & SELENBP1 & $-46 \%$ & KIF3B & $38 \%$ \\
\hline CIRBP & $-85 \%$ & CSF1R & $-62 \%$ & ATP5B & $-38 \%$ & PKM2 & $38 \%$ \\
\hline FOXO1 & $-85 \%$ & GAS6 & $-62 \%$ & AXL & $-38 \%$ & RFC4 & $38 \%$ \\
\hline ITGB3 & $-85 \%$ & NF2 & $-62 \%$ & CTNNA1 & $-38 \%$ & RARA & $46 \%$ \\
\hline PTEN & $-85 \%$ & $\mathrm{PECl}$ & $-62 \%$ & DCN & $-38 \%$ & RAD21 & $54 \%$ \\
\hline RHOB & $-85 \%$ & PRKCE & $-62 \%$ & EXT1 & $-38 \%$ & PRC1 & $77 \%$ \\
\hline TYRO3 & $-85 \%$ & STAT3 & $-62 \%$ & HRB & $-38 \%$ & SKIL & $77 \%$ \\
\hline ABL1 & $-77 \%$ & TAF1 & $-62 \%$ & PPP2R5A & $-38 \%$ & & \\
\hline CD59 & $-77 \%$ & TJP1 & $-62 \%$ & PRKD2 & $-38 \%$ & & \\
\hline
\end{tabular}

List of the 102 genes from the OpenArray analysis and percentage of tumor tissues they were differentially expressed in. Negative numbers indicate under-expression. doi:10.1371/journal.pone.0009122.t003

The difference between these and the remaining 31 ipsilateral tissues cannot be correlated with tissue or patient characteristics (supplemental Tables S1 and S2). Because BRCA status was not recorded, the study does not address any possible link between mutation and a cancer-like gene expression pattern in ipsilateral normal tissue. Another explanation is a positional effect related to distance between lesion and the site of normal tissue collection or that index lesion and ipsilateral normal tissue come from the same lobe [38]. Consequently, a normal tissue from an unaffected contralateral breast should display a normal-like gene expression pattern. Indeed, of the three contralateral normal tissues, the two coming from a breast without evidence of cancer were found in the normal cluster and the third, from a breast with malignancy, grouped with the cancers.

Tripathi et al. compared normal tissue from mammaplasty to ipsilateral normal and breast tissue with in situ disease. They found that global gene expression abnormalities exist in both normal epithelium of breast cancer patients and early cancers [6]. The results presented here go one step further by including samepatient invasive tissues. This leads to the conclusion that ipsilateral normal tissues with cancer-like gene expression are molecularly predisposed to cancer. To validate these findings, BRCA status of the patient and positional information of the tissue pieces harvested from a breast would need to be recorded. Our tissue collection protocol has been altered accordingly.

\section{Conclusions}

Our results suggest that many of the genes commonly attributed to cancer pathways are expressed at lower levels in breast cancers than in normal breast tissue, confirming and further extending results by Tripathi et al. [6]. Furthermore, the genes that predict aggressive phenotype in between-cancer comparisons are in their majority not differentially expressed between aggressive cancers and healthy controls. If serum assays commonly measure the increase of a marker rather than its absence in cancer, our findings would help explain the current lack of suitable blood markers for breast cancer, particularly in patients with poor prognosis malignancies. With the exception of three genes (MMP12, $S 100 A 7$ and SPPI) whose expression requires further validation, we were unable to find markers with potential utility for the detection of aggressive disease.

In spite of these shortcomings, our work resulted in the identification of a number of differentially expressed genes, including a minimum of 46 discriminating between cancer and controls and 12 related to aggressive disease. These 46 also identify regions of ipsilateral histologically 'normal' breast tissue are predisposed to becoming malignant. Of all discovered genes, those coding for proteins that are readily shed may be of interest for serum marker evaluation, assuming a positive correlation between transcript and protein expression and that the proteins are shed into the blood. Markers that are over-expressed but not 
shed may be the most attractive for tumor-specific localization, including prognosis.

\section{Methods}

\section{Ethics Statement}

This study was conducted according to the principles expressed in the Declaration of Helsinki. The study was approved by the Institutional Review Board of Fred Hutchinson Cancer Research Center (IRB\#5317), Gedars Sinai Cancer Center (IRB\#4321/ CR00002187) and Swedish Medical Center (IRB\#3992C-03). All patients provided written informed consent for the collection of samples and subsequent analysis.

\section{Patients and Tissues}

Patients were enrolled at Swedish Medical Center, Seattle and Cedars Sinai Medical Center, Los Angeles. Patients were consented before surgery and administered a health status and family history questionnaire. Hospital records were used for follow-up. Patient characteristics are reported in the supplemental Table S1. Cancer, ipsi- and contralateral normal tissues were obtained at Swedish Medical Center from 44 patients (including 7 with neoadjuvant treatment) undergoing mastectomy. Tissues from breast reduction surgeries (20 patients) were obtained from private practices in Los Angeles and histologically analyzed to exclude any with abnormalities. In all cases, tissue was obtained and processed by research personnel in the operating or gross room and frozen within 1 hour of surgery. The frozen tissue available for research (mean: $150 \mathrm{~g}$, range: $20-500 \mathrm{~g}$ ) was split into several pieces of which one was fixed in formalin, embedded in paraffin and used for histological examination by a pathologist. The other pieces were kept frozen and used for RNA extraction. No microdissection was performed. Viable tumor cell content in the cancer tissues varied from $15 \%$ to $95 \%$, the rest being mostly fibrous and fatty tissue (Supplemental Table S2). We did not select tissues with more than $60 \%$ fat content. The variability in tumor cell volume is a consequence of our collection procedure where samples were taken from various distances to the main tumor mass. Furthermore, lobular carcinomas (25\% of our cancer tissues) often display low tumor cellularity. Unsupervised cluster analysis of these tissues showed no cluster formation based on tumor cell content (Supplemental Figure S2). In the end, gross and microscopic clinical evaluation matched the histology of the actual tissue piece being analyzed in $50 \%$ of the cancer tissues and $67 \%$ of tissues with normal histology.

\section{LevelsDB}

Over the last 10 years a database has been compiled (LevelsDB) that holds gene and protein expression information from over 134 publications (90\% transcript-, $10 \%$ protein-based) and 21,890 genes. LevelsDB was created to facilitate the discovery of markers for cancer detection, and emphasis was given to publications with data for normal controls as well as cancers. LevelsDB uses the GeneID as an identifier [39] which is related to the gene symbols governed by the Guidelines for Human Gene Nomenclature [40]. The datasets were extremely variable in the way they recorded expression, ranging from a simple list of proteins to raw cDNA microarray expression data. As a consequence, LevelsDB forewent exact representation of original expression values. Instead, it recorded whether or not a transcript or protein was present in a given tissue, whether it had tumor-to-normal ratios above a factor 2 or, in the case of cDNA microarray-based expression data, whether a mRNA was expressed at low, medium or high levels (threshold defined by $1 \mathrm{x}, 3 \mathrm{x}$, and 10x the median expression across all tissues). LevelsDB also contains data on subcellular localization.
The datasets used in LevelsDB are listed in the supplemental Document S1. Access to this database is available upon request.

\section{RNA Extraction and Real-Time PCR (SYBR)}

Snap-frozen tissues were homogenized with a TissueLyser (Qiagen, Valencia, CA) in Trizol (Invitrogen, Carlsbad, CA). Total RNA was then extracted using RNeasy with DNAse I (Qiagen). RNA quality was measured by Agilent 2100 Bioanalyzer (Agilent, Santa Clara, CA) to have a 28S/18S RNA ratio of $1 \pm 0.2$ and by spectrophotometer with an $\mathrm{OD}_{260} / \mathrm{OD}_{280}$ ratio $>1.6$ ). Mean total RNA yield was $90 \pm 130 \mu \mathrm{g}$ per mg of tissue. Copy DNA was reverse transcribed from $5 \mu \mathrm{g}$ of total RNA (Superscript III kit, Invitrogen) with oligo-dT priming, of which $50 \mathrm{ng}$ were used as template in a $15 \mu \mathrm{l} \mathrm{PCR}$. Copy DNA was amplified using the SYBR green kit (Invitrogen) on a 7900HT Fast Real-Time PCR System (Applied Biosystems, Foster City, CA). Each 384-well plate contained aliquots of all cDNAs used during the experiment as well as a standard made with testes cDNA in 5 dilutions $(1: 1$, 1:3, 1:9, 1:27, 1:81) as duplicates, amplified with primers for $A C T B$ (all cDNAs were sub-aliquoted and stored at $-20^{\circ} \mathrm{C}$ for consistency). This allowed us to transform the logarithmic cycle threshold (CT) values into linear values. Reactions were performed in duplicate or, if samples did not amplify well or if the correlation between the runs was poor, in triplicate. All PCRs were normalized by the averaged expression of three housekeeping genes $A C T B, B 2 M$ and TMED10 run in triplicate. Primer sequences are listed in the supplemental Document S3.

\section{OpenArray Transcript Expression}

Two micrograms of total RNA were reverse transcribed using the High Capacity cDNA RT Kit (Applied Biosystems) with random hexamer primers. All cDNA was analyzed on the Cancer Pathways OpenArray system (BioTrove, Woburn, MA) using the Fast Start DNA SYBR Green kit (Roche, Nutley, NJ). Four cDNA samples were tested simultaneously per plate, with 16 samples per run. CT values were transformed into linear values by calculating $1.735^{\wedge}(32-\mathrm{CT})$. Values were normalized by the mean of 18 housekeeping genes. The expression values from the OpenArray platform and the qPCR (SYBR) were mean-normalized to allow for comparison across the two platforms.

\section{Cluster Analysis}

Unsupervised hierarchical clustering was performed using Spearman rank correlation as similarity metric and centroid linkage as clustering method. PCR expression values were averaged between duplicate runs, mean-normalized and entered into the Cluster program [41] as $\log 2$ values. The tree was visualized using Java Treeview [42].

\section{Supporting Information}

Document S1 List of the datasets used in LevelsDB and references for the 134 potential marker genes.

Found at: doi:10.1371/journal.pone.0009122.s001 (0.06 MB RTF)

Document S2 PCR expression data of the 134 genes in 93 tissues including explanation of the data processing.

Found at: doi:10.1371/journal.pone.0009122.s002 (0.06 MB CSV)

Document S3 Primer sequences for the genes amplified the qPCR.

Found at: doi:10.1371/journal.pone.0009122.s003 (0.01 MB CSV) 
Table S1 Characteristics of the 64 patients who donated tissue. Age at diagnosis, Diagnosis (IDC = invasive ductal carcinoma, $\mathrm{ILC}=$ invasive lobular carcinoma, $\mathrm{MET}=$ metaplastic carcinoma, $\mathrm{INF}=$ inflammatory carcinoma, $\mathrm{MUC}=$ mucinous carcinoma, $\mathrm{NML}=$ normal), Stage, grade and tumor size noted during gross histopathological examination, Number of positive lymph nodes out of all tested, BI-RADS mammography score ( $1=$ normal, $2=$ benign, $3=$ probably benign, $4=$ suspicious, $5=$ malignant $)$, Breast Density score $(0=$ fatty, $1=$ average, $2=$ dense, $3=$ very dense), Race (White, Black, Hispanic, Asian), Hormone receptor (estrogen and/or progesterone) and ERBB2 status, and the tissue(s) used $(\mathrm{NI}=$ normal ipsilateral, $\mathrm{CA}=$ invasive cancer, $\mathrm{NC}=$ normal contralateral, $\mathrm{NM}=$ normal from mammaplasty; fatty $=20-50 \%$ fat). Progression-free survival (PFI) and survival given in months. $\mathrm{NED}=$ no evidence of disease. Empty cells: no information. It should be noted that the breast reduction population was 15 years younger than the breast cancer population.

Found at: doi:10.1371/journal.pone.0009122.s004 (0.47 MB PDF)

Table S2 Tissue Characteristics. Histopathological examination was performed on the tissue piece adjacent to the one used for RNA extraction. Patient numbers in the first column match those in table $\mathrm{S} 1$. Tissue classification is explained in table $\mathrm{S} 1$. In the case of tumor tissue, the histology lists the percentage of the tissue specimen that was involved by tumor. The percentage of viable tumor cells, necrosis and the inflammatory component within the tumor histology is listed in the last three columns. These numbers donE $1 / 4 \mathrm{t}$ add up to $100 \%$ as we did not record the percentage of fibrous or fatty tissue. However, we limited collection of all tissues to those with less than $60 \%$ fat. Please note that tumor grade of an individual tissue piece might differ from the grade of the entire tumor listed in table $\mathrm{S} 1$. Found at: doi:10.1371/journal.pone.0009122.s005 (0.31 MB PDF)

Table S3 List of the 134 genes (by gene symbol) found by mining of expression data and/or LevelsDB mining (asterisk). References are listed in the Supplement. The 8 genes for which PCR was inconclusive are struck through. Thresholds for the cancers and controls were determined by the expression in the 28 normal mammaplasty tissues as mean +3 SD for genes with overexpression in the cancers and as below the minimum for genes with under-expression in the cancers. These cutoff values lie well above the gene expression variability in normal breast tissues listed in Table 1. Columns 2 and 3 list the percent of cancer or control tissues above or below this threshold. Result column: over- or under-expression in cancer tissue for genes with $>=20 \%$ of the cancers and $<=5 \%$ of the controls above or below threshold.

Found at: doi:10.1371/journal.pone.0009122.s006 (0.06 MB PDF)

Table S4 Comparison of PCR results from the original PCR and the OpenArray results from the reduced tissue and gene set. Listed are the expression values for the tumors (pink and orange shading in the leftmost column) and the mammaplasty normal controls (green shading) for both the original (TOR) and the OpenArray platform (BT). The yellow- and purple-shaded cells represent genes with over- and under-expression respectively. Due to the extreme low number of control tissues (9), values with high

\section{References}

1. Schummer M, Ng W, Bumgarner R, Nelson P, Schummer B, et al. (1999) Comparative hybridization of an array of 21,500 ovarian cDNAs for the discovery of genes overexpressed in ovarian carcinomas. Gene 238: 375-385.

2. Pepe MS, Etzioni R, Feng Z, Potter JD, Thompson ML, et al. (2001) Phases of biomarker development for early detection of cancer. J Natl Cancer Inst 93: 1054-1061. error (outliers) have a huge influence on the cutoff and hence the overall results. Four of these outliers were removed (empty cells). Found at: doi:10.1371/journal.pone.0009122.s007 (0.25 MB PDF)

Table S5 Percent CV values of original and unfiltered OpenArray PCR results. Percent $\mathrm{CV}$ values for all tissues (\%CV a) and for only those tissues with outliers were removed from the OpenArray results ( $\% \mathrm{CV}$ b). Removal was acceptable because the OpenArray PCR was not duplicated like the original PCR and had a higher number of values close or below the PCRE $1 / 4$ s detection limit.

Found at: doi:10.1371/journal.pone.0009122.s008 (0.04 MB PDF)

Table S6 Composition of the Sub-Cluster of the Cancer Cluster. Shown are the number of patients and the percentage of the total number of patients per cluster. Highlighted are the features that give the patients in the luminal-enriched cluster a better prognosis. Found at: doi:10.1371/journal.pone.0009122.s009 (0.03 MB PDF)

Figure S1 Unsupervised cluster analysis of the filtered OpenArray data (112 genes, 13 invasive cancers and 9 normal control tissues). The OpenArray dataset contains a high proportion of genes with under-expression in the invasive cancer tissues (pink shading on the left) compared to the healthy controls (green shading on the left). The genes found to be under- or overexpressed by the original dataset are indicated by a green or red dot next to their names (right). These are found in the expected cluster, further confirming the original PCR results. The tissue descriptor above lists the histology (IDC in pink, ILC and IDLC in orange and NML in green) followed by the tissue identifier.

Found at: doi:10.1371/journal.pone.0009122.s010 (0.23 MB PDF)

Figure S2 Unsupervised cluster analysis of the 24 invasive cancer tissues ( 7 tissues with $>=60 \%$ and 17 with $<=30 \%$ viable tumor cells) using the 67 genes that discriminate between invasive tissues and mam- maplasty normal tissues. No cluster formation is observed based on tumor cell content. The two clusters that are formed separate the cancers by outcome.

Found at: doi:10.1371/journal.pone.0009122.s011 (0.24 MB PDF)

\section{Acknowledgments}

We would like to thank the Swedish Cancer Institute surgeons Patricia Dawson, Claire Buchanan, James Hanson, Christine Lee and Darlene Barr and the pathologists at Cellnetix, in particular Ron Tickman and Nan Ping Wang. We would also like to thank Christopher Miller and Anthony Blau for providing the EPO and EPOR primers, Shirley Gough for her chart reviews, Mary Atwood for providing data from the Breast Cancer Research Database at the Swedish Cancer Institute and Max Drescher for his help with the PCR.

\section{Author Contributions}

Conceived and designed the experiments: MS NDU. Performed the experiments: AG JG. Analyzed the data: MS AG MWM. Contributed reagents/materials/analysis tools: JDB BYK SK JG ST. Wrote the paper: MS JDB BYK SK MWM NDU.

3. Hellström I, Raycraft J, Hayden-Ledbetter M, Ledbetter J, Schummer M, et al. 2003) The HE4 (WFDC2) protein is a biomarker for ovarian carcinoma. Cancer Res 63: 3695-3700.

4. Scholler N, Crawford M, Sato A, Drescher C, O'Briant K, et al. (2006) BeadBased ELISA for Validation of Ovarian Cancer Early Detection Markers. Clin Cancer Res 12: 2117-2124. 
5. Duffy MJ (2006) Serum tumor markers in breast cancer: are they of clinical value? Clin Chem 52: 345-351.

6. Tripathi A, King G, de la Morenas A, Perry VK, Burke B, et al. (2008) Gene expression abnormalities in histologically normal breast epithelium of breast cancer patients. Int J Cancer 122: 1557-1566.

7. Perou CM, Sørlie T, Eisen MB, van de Rijn M, Jeffrey SS, et al. (2000) Molecular portraits of human breast tumours. Nature 406: 747-752.

8. Dressman MA, Walz TM, Lavedan C, Barnes L, Buchholtz S, et al. (2001) Genes that co-cluster with estrogen receptor alpha in microarray analysis of breast biopsies. Pharmacogenomics J 1: 135-141.

9. Sørlie T, Perou CM, Tibshirani R, Aas T, Geisler S, et al. (2001) Gene expression patterns of breast carcinomas distinguish tumor subclasses with clinical implications. Proc Natl Acad Sci U S A 98: 10869-10874.

10. Sørlie T, Tibshirani R, Parker J, Hastie T, Marron JS, et al. (2003) Repeated observation of breast tumor subtypes in independent gene expression data sets. Proc Natl Acad Sci U S A 100: 8418-8423.

11. Zhao H, Langerod A, Ji Y, Nowels KW, Nesland JM, et al. (2004) Different gene expression patterns in invasive lobular and ductal carcinomas of the breast. Mol Biol Cell 15: 2523-2536.

12. Amatschek S, Koenig U, Auer H, Steinlein P, Pacher M, et al. (2004) Tissuewide expression profiling using cDNA subtraction and microarrays to identify tumor-specific genes. Cancer Res 64: 844-856.

13. Abba MC, Drake JA, Hawkins KA, Hu Y, Sun H, et al. (2004) Transcriptomic changes in human breast cancer progression as determined by serial analysis of gene expression. Breast Cancer Res 6: R499-513.

14. Turashvili G, Bouchal J, Baumforth K, Wei W, Dziechciarkova M, et al. (2007) Novel markers for differentiation of lobular and ductal invasive breast carcinomas by laser microdissection and microarray analysis. BMC Cancer 7: 55.

15. Grigoriadis A, Mackay A, Reis-Filho JS, Steele D, Iseli C, et al. (2006) Establishment of the epithelial-specific transcriptome of normal and malignant human breast cells based on MPSS and array expression data. Breast Cancer Res 8: R56.

16. Covell DG, Wallqvist A, Rabow AA, Thanki N (2003) Molecular classification of cancer: unsupervised self-organizing map analysis of gene expression microarray data. Mol Cancer Ther 2: 317-332.

17. Crean PA, Pratt T, Davies GJ, Myers M, Lavender P, et al. (1986) The fractional distribution of the cardiac output in man using microspheres labelled with technetium 99m. Br J Radiol 59: 209-215.

18. Strauss HW, Harrison K, Pitt B (1977) Thallium-201: non-invasive determination of the regional distribution of cardiac output. J Nucl Med 18: 1167-1170.

19. Lantz BM, Foerster JM, Link DP, Holcroft JW (1981) Regional distribution of cardiac output: normal values in man determined by video dilution technique. AJR Am J Roentgenol 137: 903-907.

20. Shyamsundar R, Kim YH, Higgins JP, Montgomery K, Jorden M, et al. (2005) A DNA microarray survey of gene expression in normal human tissues. Genome Biol 6: R22.

21. Morrison T, Hurley J, Garcia J, Yoder K, Katz A, et al. (2006) Nanoliter high throughput quantitative PCR. Nucleic Acids Res 34: e123.

22. Köhrmann A, Kammerer U, Kapp M, Dietl J, Anacker J (2009) Expression of matrix metalloproteinases (MMPs) in primary human breast cancer and breast cancer cell lines: New findings and review of the literature. BMC Cancer 9: 188.
23. Bièche I, Lerebours F, Tozlu S, Espie M, Marty M, et al. (2004) Molecular profiling of inflammatory breast cancer: identification of a poor-prognosis gene expression signature. Clin Cancer Res 10: 6789-6795.

24. Srour N, Reymond MA, Steinert R (2008) Lost in translation? A systematic database of gene expression in breast cancer. Pathobiology 75: 112-118.

25. Charpin C, Giusiano S, Secq V, Carpentier S, Andrac L, et al. (2009) Quantitative immunocytochemical profile to predict early outcome of disease in triple-negative breast carcinomas. Int J Oncol 34: 983-993.

26. Jacquemier J, Padovani L, Rabayrol L, Lakhani SR, Penault-Llorca F, et al. (2005) Typical medullary breast carcinomas have a basal/myoepithelial phenotype. J Pathol 207: 260-268.

27. Nielsen TO, Hsu FD, Jensen K, Cheang M, Karaca G, et al. (2004) Immunohistochemical and clinical characterization of the basal-like subtype of invasive breast carcinoma. Clin Cancer Res 10: 5367-5374.

28. Fabre-Lafay S, Monville F, Garrido-Urbani S, Berruyer-Pouyet C, Ginestier C, et al. (2007) Nectin-4 is a new histological and serological tumor associated marker for breast cancer. BMC Cancer 7: 73.

29. Narita D, Raica M, Suciu C, Cimpean A, Anghel A (2006) Immunohistochemical expression of androgen receptor and prostate-specific antigen in breast cancer. Folia Histochem Cytobiol 44: 165-172.

30. Pestalozzi BC, Zahrieh D, Mallon E, Gusterson BA, Price KN, et al. (2008) Distinct clinical and prognostic features of infiltrating lobular carcinoma of the breast: combined results of 15 International Breast Cancer Study Group clinical trials. J Clin Oncol 26: 3006-3014.

31. Zhao H, Langerød A, Ji Y, Nowels KW, Nesland JM, et al. (2004) Different gene expression patterns in invasive lobular and ductal carcinomas of the breast. Mol Biol Cell 15: 2523-2536.

32. Tlsty TD (2007) Luminal cells GATA have it Nat Cell Biol 9: 135-136.

33. Dian D, Herold H, Mylonas I, Scholz C, Janni W, et al. (2009) Survival analysis between patients with invasive ductal and invasive lobular breast cancer. Arch Gynecol Obstet 279: 23-28.

34. Sivridis E, Stamos C, Fiska A, Nikolettos N, Koukourakis MI, et al, (2009) cerbB-2 and the "triple-state" in early breast carcinomas. Med Oncol 23: 23.

35. Oh DS, Troester MA, Usary J, Hu Z, He X, et al. (2006) Estrogen-regulated genes predict survival in hormone receptor-positive breast cancers. J Clin Oncol 24: $1656-1664$.

36. Paik S, Shak S, Tang G, Kim C, Baker J, et al. (2004) A multigene assay to predict recurrence of tamoxifen-treated, node-negative breast cancer. N Engl J Med 351: 2817-2826.

37. van 't Veer LJ, Dai H, van de Vijver MJ, He YD, Hart AA, et al. (2002) Gene expression profiling predicts clinical outcome of breast cancer. Nature 415: 530-536.

38. Tot T (2007) The theory of the sick breast lobe and the possible consequences. Int J Surg Pathol 15: 369-375.

39. Maglott D, Ostell J, Pruitt KD, Tatusova T (2005) Entrez Gene: gene-centered information at NCBI. Nucleic Acids Res 33: D54-58.

40. Wain HM, Bruford EA, Lovering RC, Lush MJ, Wright MW, et al. (2002) Guidelines for human gene nomenclature. Genomics 79: 464-470.

41. Eisen MB, Spellman PT, Brown PO, Botstein D (1998) Cluster analysis and display of genome-wide expression patterns. Proceedings of the National Academy of Sciences of the United States of America 95: 14863-14868.

42. Saldanha AJ (2004) Java Treeview-extensible visualization of microarray data. Bioinformatics 20: 3246-3248. 